\title{
Graphs of low chordality
}

\author{
L.S. Chandran ${ }^{1}$ and Vadim V. Lozin ${ }^{2}$ and C.R. Subramanian ${ }^{3}$ \\ ${ }^{1}$ Max-Planck Institute for Informatik, Stuhlsatzenhausweg 85, 66123 Saarbrücken, Germany. \\ Email: sunil@mpi-sb.mpg.de \\ ${ }^{2}$ RUTCOR, Rutgers University, 640 Bartholomew Rd., Piscataway, NJ 08854-8003. \\ Email: lozin@rutcor.rutgers.edu \\ ${ }^{3}$ The Institute of Mathematical Sciences, Chennai, 600113, India. \\ Email: crseimsc.res.in
}

received Mar 20, 2004, revised Feb 7, 2005, accepted Mar 28, 2005.

The chordality of a graph with at least one cycle is the length of the longest induced cycle in it. The odd (even) chordality is defined to be the length of the longest induced odd (even) cycle in it. We show that co-circular-arc graphs and co-circle graphs have even chordality at most 4 . We also identify few other classes of graphs having bounded (by a constant) chordality values.

Keywords: induced cycles, chordality

\section{Introduction}

The chordality of an undirected graph $G$, which is not acyclic, is defined as the length of the longest induced cycle in it. The chordality of an acyclic graph is defined to be 0 . We use $C_{l}(l \geq 3)$ to denote a cycle of length $l$. An induced cycle is called a hole. A hole is an odd hole if its length is odd and is an even hole otherwise. Odd-chordality of a graph is the length of the longest odd hole in it. Even-chordality of a graph is the length of the longest even hole in it. In the present paper we identify several classes of graphs of bounded chordality. Our motivation is due to some recent interesting results connecting chordality with other structural aspects of graphs. We list some of them below.

1. Bodlaender and Thilikos [3] show that if a graph has chordality at most $k$ and maximum degree at most $\Delta$, then its treewidth is at most $\Delta(\Delta-1)^{k-3}$. (For the definition of treewidth and for a brief review of its applications, both theoretical and practical, see [2].)

2. In the same paper mentioned above, Bodlaender and Thilikos [3] prove some separator theorems for graphs of low chordality.

3. In a recent work, Chandran and Ram [5] relate the chordality with the number of minimum cuts in a graph (with positive edge weights). They show that if the chordality of a graph with $n$ nodes is at most $k$, then the number of minimum cuts possible in that graph is at most $\frac{(k+1) n}{2}-k$, irrespective of the weight function as long as the weights are positive. 
4. Chandran and Subramanian [6] relate the second smallest eigen-value $\mu$ of the Laplacian matrix of the graph to its chordality. They show that if the chordality of an $n$ node graph is at most $k$ and the maximum degree is at most $\Delta$, then $\mu \leq \frac{8 \Delta^{k-1}}{n}$

5. Chepoi and Dragan [4] show that for any connected graph $G$ of chordality at most $k$ there exists a tree $T$ on the same vertex set such that $\left|d_{G}(u, v)-d_{T}(u, v)\right| \leq\lfloor k / 2\rfloor+\alpha$ for any pair of vertices $u$ and $v$, where $d(u, v)$ is the distance between $u$ and $v$, and $\alpha$ is a constant $(\alpha=1$ if the chordality is either 4 or 5 and $\alpha=2$ otherwise).

6. F. Dragan [9] proposes a very simple and efficient approach to solve the all pairs shortest path and all pairs almost shortest path problems on graphs of low chordality.

7. F. Gavril [12] presents an algorithm that finds a maximum weight induced path in a graph with $n$ vertices, $m$ edges and of chordality at most $k$ in time $O\left(m n^{k}\right)$. In general the problem is known to be NP-hard.

8. The Strong Perfect Graph Theorem recently proved by Chudnovsky, Robertson, Seymour and Thomas [7], asserts that a graph $G$ is perfect if and only if the odd chordality of $G$ and its complement is at most 3 .

Many well-known graph classes have bounded chordality. For instance, it follows directly from the definition that chordal graphs (those having no holes of length 4 or more) have chordality at most 3 , and weakly chordal graphs (those having neither holes of length at least 5 nor their complements) have chordality at most 4 . Deimer proved in $[8]$ that the chordality of a $d$-dimensional hypercube is at most $2^{d-1}\left(1-1 /\left(d^{2}-5 d+7\right)\right)$ for $d \geq 7$. It would be of interest to identify other classes of graphs of bounded chordality.

Our main result is a proof of boundedness of even-chordality of co-circular-arc and co-circle graphs. In addition, we also identify few other classes having bounded chordality values.

For each class, in addition to deriving bounds on their chordality values, we also provide examples to show that these bounds are tight.

All graphs considered in this paper are finite, simple and undirected. For a graph $G$, we denote by $V(G)$ and $E(G)$ its vertex set and edge set, and by $\bar{G}$ the complement of $G$. As usual, $P_{n}$ and $K_{n}$ denote a chordless path and a complete graph with $n$ vertices, respectively. Also, $G+H$ stands for the disjoint union of two graphs $G$ and $H$. In particular, $m G$ is the disjoint union of $m$ copies of $G$. For a class $C$ of graphs, we use co- $\mathcal{C}$ to denote the class of complements of members of $\mathcal{C}$.

\section{Co-circular-arc graphs and co-circle graphs}

The main result of this section is the following theorem.

Theorem 2.1 For each graph that is the complement of either a circular-arc graph or a circle graph, its even-chordality is at most 4 while there is no upper bound on its odd-chordality.

Below, we prove Theorem 2.1 by looking at each of the classes mentioned and providing justifications. 
Co-circular-arc graphs: These are complements of circular-arc graphs. A circular-arc graph is the intersection graph of the arcs on the circumference of a unit circle. Co-circular-arc graphs have evenchordality at most 4 and this class has no bound on their odd-chordality since for each $k \geq 1$, the induced $C_{2 k+1}$ is co-circular-arc.

Before we see the proof of this result, we introduce a convention:

Direct each arc on the circumference of the unit circle according to the clockwise direction. Now each arc on the circumference is specified by an ordered pair $(b, e)$ where $b$ (respectively $e$ ) denotes the angle $\phi \in[0,2 \pi)$ that the beginning point (respectively the ending point) of this directed arc makes with the positive part of the $x$-axis. The angle increases in the clockwise direction. It is possible that $b>e$.

Let $G$ be a co-circular-arc graph. Let $v \rightarrow A(v)=\left(b_{v}, e_{v}\right)$ be the mapping of $V(G)$ onto circular-arcs such that $u, v \in V(G)$ are neighbors (in $G$ ) if and only if $A(u)$ and $A(v)$ have empty intersection. First, we prove that $G$ has even-chordality at most 4 .

Claim 1 We can assume, without loss of generality, that no arc $A(u)$ properly contains any other arc $A(v)$.

Proof: To see this, consider any induced $C_{l}, l \geq 5$, in $G$ and consider any two distinct vertices $a, b$ in $C_{l}$. We can always find (since $l \geq 5$ ) two distinct vertices $c, d$ on $C_{l}$ such that $a$ is a neighbor of $c$ but not a neighbor of $d$ and $b$ is a neighbor of $d$ but not a neighbor of $c$. If, say, $A(a) \subset A(b)$, it implies $A(d) \cap A(b) \neq \emptyset$ and $d$ is not a neighbor of $b$. Similarly, we cannot have $A(b) \subset A(a)$. Hence, we can assume that no arc properly contains any other arc.

Claim 2 For any induced path $x-0-1-\ldots-l$ in $G$ with $b_{0}=0, b_{1}<b_{x}$, the endpoints of the arcs $\{A(x), A(0), \ldots, A(l)\}$ should appear according to the following increasing sequence $\sigma_{l}$ :

If $l=2 k$,

$$
\begin{aligned}
& 0, e_{2 k}, e_{2 k-2}, b_{2 k-1}, e_{2 k-4}, b_{2 k-3}, \ldots, e_{2}, b_{3}, e_{0}, b_{1}, \\
& b_{x}, e_{2 k-1}, b_{2 k}, e_{2 k-3}, b_{2 k-2}, \ldots, e_{3}, b_{4}, e_{1}, b_{2}, e_{x}, 2 \pi
\end{aligned}
$$

If $l=2 k+1$,

$$
\begin{gathered}
0, e_{2 k}, b_{2 k+1}, e_{2 k-2}, b_{2 k-1}, \ldots, e_{2}, b_{3}, e_{0}, b_{1}, \\
b_{x}, e_{2 k+1}, e_{2 k-1}, b_{2 k}, e_{2 k-3}, b_{2 k-2}, \ldots, e_{3}, b_{4}, e_{1}, b_{2}, e_{x}, 2 \pi
\end{gathered}
$$

Proof: We prove this by induction on $k$ where either $l=2 k$ or $l=2 k+1$.

The base cases $l=0,1,2,3$ corresponding to $k=0,1$ can be easily verified to be true.

Assume that the claim is true for all $k^{\prime} \leq k$ where $k \geq 1$.

We now prove it for $k+1$.

First, consider the induced path $x-0-1-\ldots-2 k+2$. The endpoints of $\{x, 0,1, \ldots, 2 k+1\}$ should appear according to $\sigma_{2 k+1}$. Since 0 and $2 k+2$ are not neighbors in $G, A(0)$ and $A(2 k+2)$ have non-empty intersection. Hence either $b_{2 k+2}$ or $e_{2 k+2}$ (but not both) should lie between $0=b_{0}$ and $e_{0}$.

If $b_{2 k+2}$ lies in $\left(0, e_{0}\right)$, then $e_{2 k+2}$ should come after $e_{0}$. This implies that $A(2 k+1)$ and $A(2 k+2)$ have non-empty intersection violating the assumption that $2 k+1$ and $2 k+2$ are neighbors in $G$.

Hence only $e_{2 k+2}$ lies in $A(0)$. In that case, the arc corresponding to the segment $\left[0, e_{2 k+2}\right]$ lies within $A(2 k+2)$. Also, $e_{2 k+2}$ should come before $e_{2 k}$. To see this, suppose it comes after $e_{2 k}$. It certainly cannot come after $b_{2 k+1}$ since $A(2 k+1)$ and $A(2 k+2)$ have empty intersection. 
Hence $e_{2 k+2}$ lies between $e_{2 k}$ and $b_{2 k+1}$. Hence $b_{2 k+2}$ comes after $b_{2 k}$ but before $e_{2 k-1}$ and this is not possible since $b_{2 k}$ comes after $e_{2 k-1}$ in $\sigma_{2 k+1}$.

Thus, $e_{2 k+2}$ should come before $e_{2 k}$. Hence $b_{2 k+2}$ should come before $b_{2 k}$. Also, it should come before $e_{2 k-1}$ to ensure that $2 k-1$ and $2 k+2$ are not neighbors. Also, it should come after $e_{2 k+1}$ to ensure that $2 k+1$ and $2 k+2$ are neighbors.

Since the positions of $b_{2 k+2}$ and $e_{2 k+2}$ are forced in this way, by placing these, we see that the endpoints appear according to $\sigma_{2 k+2}$. This proves Claim 2 for $l=2 k+2$.

Similarly, one can prove Claim 2 for $l=2 k+3$ from $l=2 k+2$ by observing that $(i)$ only $b_{2 k+3}$ is in $\left(0, e_{0}\right)$, (ii) $b_{2 k+3}$ should lie between $e_{2 k+2}$ and $e_{2 k}$, (iii) $e_{2 k+3}$ should lie between $b_{x}$ and $e_{2 k+1}$. This proves Claim 2 .

Now consider any induced cycle $C_{s}$ of even length $s \geq 6$ in $G$. Without loss of generality, by rotating the unit circle around its centre, we can assume that $(i)$ there exists a vertex 0 on $C_{s}$ with $b_{0}=0$, (ii) if 1 and $x$ are the neighbors of 0 in $G$, then $b_{1}<b_{x}$. Thus, we can assume that $C_{s}=(x, 0,1, \ldots, 2 k, x)$ where $k \geq 2$. Now $x-0-1-\ldots-(2 k-1)$ is an induced path and hence, by Claim 2 , the corresponding endpoints should appear according to $\sigma_{2 k-1}$ as given below.

$$
\begin{gathered}
0, e_{2 k-2}, b_{2 k-1}, e_{2 k-4}, b_{2 k-3}, \ldots, e_{2}, b_{3}, e_{0}, b_{1}, \\
b_{x}, e_{2 k-1}, e_{2 k-3}, b_{2 k-2}, e_{2 k-5}, b_{2 k-4}, \ldots, e_{3}, b_{4}, e_{1}, b_{2}, e_{x}, 2 \pi
\end{gathered}
$$

Since $2 k$ is a neighbor of both $x$ and $2 k-1$, we should have $A(2 k) \cap(A(x) \cup A(2 k-1))=\emptyset$. But, this implies that $A(2 k) \cap A(1)=\emptyset$ since $A(1) \subset(A(x) \cup A(2 k-1))$ as can be seen from $\sigma_{2 k-1}$. This is not possible since 1 and $2 k$ are not neighbors and hence $A(1)$ and $A(2 k)$ should have non-empty intersection. This shows that even-chordality of co-circular-arc graphs is at most 4 .

This bound is tight because of the following example. Consider the set of arcs

$$
A(0)=(0, \pi / 2), A(1)=(3 \pi / 4,5 \pi / 4), A(x)=(\pi, 3 \pi / 2), A(2)=(7 \pi / 4, \pi / 4)
$$

Induced $C_{4}$ is the complement of the intersection graph of these arcs.

Surprisingly, there is no bound on the odd-chordality of co-circular-arc graphs and for every $k \geq 0$, induced $C_{2 k+3}$ is co-circular arc. To see this, note that $C_{2 k+3}$ is the same as the induced path $x-0-1-\ldots-2 k+1$ except that we want, in addition, $x$ and $2 k+1$ to be neighbors. This can be made co-circular-arc by picking values for $\left(b_{x}, e_{x}\right),\left(b_{0}=0, e_{0}\right), \ldots,\left(b_{2 k+1}, e_{2 k+1}\right)$ so that, after sorting, these values appear as in the following sequence (which is obtained from $\sigma_{2 k+1}$ by moving $e_{2 k+1}$ to a position between $b_{1}$ and $b_{x}$ ):

$$
\begin{gathered}
0, e_{2 k}, b_{2 k+1}, e_{2 k-2}, b_{2 k-1}, \ldots, e_{2}, b_{3}, e_{0}, b_{1}, \\
e_{2 k+1}, b_{x}, e_{2 k-1}, b_{2 k}, e_{2 k-3}, b_{2 k-2}, \ldots, e_{3}, b_{4}, e_{1}, b_{2}, e_{x}, 2 \pi
\end{gathered}
$$

One can pick values so as to appear like this. This shows that each odd hole is a co-circular-arc graph. Note that each hole (odd or even) is also a circular-arc graph.

Co-circle graphs: These are complements of circle graphs. A circle graph is the intersection graph of the chords of a unit circle. A chord of a circle is a straight-line segment joining two points on the circumference of the circle. Here, we assume that any two chords either have empty intersection or intersect at an internal point (not at the endpoints of the chords). As in the case of co-circular-arc graphs, 
co-circle graphs also have even-chordality at most 4 with no bound on their odd-chordality since for each $k \geq 1$, the induced $C_{2 k+1}$ is a co-circle graph.

We use the following convention for representing the chords of a circle:

Each chord of the circle is specified by an ordered pair $(b, e)$ where $b$ and $e$ denote the angle $\phi \in[0,2 \pi)$ that the two endpoints of the chord make with the $x$-axis with the convention that $b<e$. The angle increases in the clockwise direction.

Let $G$ be a co-circle graph. Let $v \rightarrow A(v)=\left(b_{v}, e_{v}\right)$ be the mapping of $V(G)$ onto chords of a unit circle such that $u, v \in V(G)$ are neighbors (in $G$ ) if and only if $A(u)$ and $A(v)$ have empty intersection. In other words, $u$ and $v$ are neighbors if and only if either $b_{u}<b_{v}<e_{v}<e_{u}$ or $b_{v}<b_{u}<e_{u}<e_{v}$ or $b_{u}<e_{u}<b_{v}<e_{v}$ or $b_{v}<e_{v}<b_{u}<e_{u}$. Equivalently, $u$ and $v$ are not neighbors if and only if either $b_{u}<b_{v}<e_{u}<e_{v}$ or $b_{v}<b_{u}<e_{v}<e_{u}$.

Claim 3 For any induced path $x-0-1-\ldots-l$ in $G$ with $b_{0}=0, b_{1}<b_{x}$, the endpoints of the chords $\{A(x), A(0), \ldots, A(l)\}$ should appear according to the following increasing sequence $\tau_{l}$ : If $l=2 k$,

$$
\begin{gathered}
0, b_{2 k}, b_{2 k-2}, b_{2 k-1}, b_{2 k-4}, b_{2 k-3}, \ldots, b_{2}, b_{3}, e_{0}, \\
b_{1}, b_{x}, e_{2 k-1}, e_{2 k}, e_{2 k-3}, e_{2 k-2}, \ldots, e_{3}, e_{4}, e_{1}, e_{2}, e_{x}, 2 \pi
\end{gathered}
$$

If $l=2 k+1$,

$$
\begin{gathered}
0, b_{2 k}, b_{2 k+1}, b_{2 k-2}, b_{2 k-1}, \ldots, b_{2}, b_{3}, e_{0}, \\
b_{1}, b_{x}, e_{2 k+1}, e_{2 k-1}, e_{2 k}, e_{2 k-3}, e_{2 k-2}, \ldots, e_{3}, e_{4}, e_{1}, e_{2}, e_{x}, 2 \pi
\end{gathered}
$$

Proof: We prove this by induction on $k$ where either $l=2 k$ or $l=2 k+1$.

The base cases $l=0,1,2,3$ corresponding to $k=0,1$ can be easily verified to be true.

Assume that the claim is true for all $k^{\prime} \leq k$ where $k \geq 1$. We now prove it for $k+1$.

First, consider the induced path $x-0-1-\ldots-2 k+2$. The endpoints of $\{x, 0,1, \ldots, 2 k+1\}$ should appear according to $\tau_{2 k+1}$.

Since 0 and $2 k+2$ are not neighbors in $G, A(0)$ and $A(2 k+2)$ have non-empty intersection. Hence $b_{2 k+2}$ should lie between $0=b_{0}$ and $e_{0}$.

Also, it should come before $b_{2 k}$.

Suppose not. Then, since $2 k$ and $2 k+2$ are not neighbors, $e_{2 k+2}$ should come after $e_{2 k}$. Now, if $b_{2 k+2}$ comes after $b_{2 k+1}$ it implies the corresponding chords have non-empty intersection violating the fact that $2 k+1$ and $2 k+2$ are neighbors in $G$.

If $b_{2 k+2}$ comes before $b_{2 k+1}$ then the chords corresponding to $2 k+2$ and $2 k-1$ have empty intersection violating the fact that $2 k+2$ and $2 k-1$ are not neighbors in $G$.

Hence $b_{2 k+2}$ should come before $b_{2 k}$. This implies that $e_{2 k+2}$ should lie between $e_{2 k+1}$ and $e_{2 k-1}$. Since the positions of $b_{2 k+2}$ and $e_{2 k+2}$ are forced in this way, by placing these, we see that the endpoints appear according to $\tau_{2 k+2}$. This proves Claim 3 for $l=2 k+2$.

Similarly, one can prove Claim 3 for $l=2 k+3$ from $l=2 k+2$ by observing that $(i) b_{2 k+3}$ should lie between $b_{2 k+2}$ and $b_{2 k}$, (ii) $e_{2 k+3}$ should lie between $b_{x}$ and $e_{2 k+1}$. This proves Claim 3 .

Now consider any induced cycle $C_{s}$ of even length $s \geq 6$ in $G$. Without loss of generality, by rotating the unit circle around its centre, we can assume that $(i)$ there exists a vertex 0 on $C_{s}$ with $b_{0}=0,(i i)$ if 1 and $x$ are the neighbors of 0 in $G$, then $b_{1}<b_{x}$. Thus, we can assume that $C_{s}=(x, 0,1, \ldots, l, x)$ where 
$l=s-2 \geq 4$ is even. Let $l-1=2 k+1$ for some $k \geq 1$. Now $x-0-1-\ldots-(l-1)$ is an induced path and hence, by Claim 3 , the corresponding endpoints should appear according to $\tau_{l-1}$.

Since $l=2 k+2$ and 0 are not neighbors we should have $0<b_{2 k+2}<e_{0}<e_{2 k+2}$. Now either $e_{2 k+2}<b_{x}$ or $e_{x}<e_{2 k+2}$, since otherwise $2 k+2$ and $x$ would not be neighbors whereas they should be. But, we cannot have $e_{x}<e_{2 k+2}$ as this would imply 1 and $2 k+2$ are not neighbors. Hence, we have $e_{2 k+2}<b_{x}$. Again, since 1 and $2 k+2$ are not neighbors and $b_{2 k+2}<b_{1}$, we should have $b_{1}<e_{2 k+2}<b_{x}$. Now, since $e_{2 k+2}<e_{2 k+1}$ and $2 k+1$ is a neighbor of $2 k+2$ in $G$, we must have $b_{2 k+1}<b_{2 k+2}$. But this would imply that $b_{2 k}<b_{2 k+2}<e_{2 k+2}<e_{2 k}$ violating $2 k$ and $2 k+1$ not being neighbors in $G$. This shows that there can be no induced $C_{s}$ in $G$ with $s-3=l-1 \geq 3$ being odd. In other words, the even-chordality of $G$ is at most 4 .

This bound is tight because of the following example. Consider the set of chords

$$
A(0)=(0, \pi / 2), A(1)=(\pi, 3 \pi / 2), A(x)=(5 \pi / 4,7 \pi / 4), A(2)=(\pi / 4,3 \pi / 4)
$$

Induced $C_{4}$ is the complement of the intersection graph of these chords.

Like in the case of co-circular-arc graphs, there is no bound on the odd-chordality of co-circle graphs and for every $k \geq 0$, induced $C_{2 k+3}$ is co-circle. This can be seen by picking values for $\left(b_{x}, e_{x}\right),\left(b_{0}=\right.$ $\left.0, e_{0}\right), \ldots,\left(b_{2 k+1}, e_{2 k+1}\right)$ so that, after sorting, these values appear as in the following sequence (which is obtained from $\tau_{2 k+1}$ by moving $e_{2 k+1}$ to a position between $b_{1}$ and $b_{x}$ ):

$$
\begin{gathered}
0, b_{2 k}, b_{2 k+1}, b_{2 k-2}, b_{2 k-1}, \ldots, b_{2}, b_{3}, e_{0}, \\
b_{1}, e_{2 k+1}, b_{x}, e_{2 k-1}, e_{2 k}, e_{2 k-3}, e_{2 k-2}, \ldots, e_{3}, e_{4}, e_{1}, e_{2}, e_{x}, 2 \pi
\end{gathered}
$$

The complement of the circle graph corresponding to this set of chords is an induced $C_{2 k+3}$. This shows that each odd hole is a co-circle graph. Also, each hole (odd or even) is a circle graph.

\section{Other classes}

Each class of this section, as well as the two classes studied above, has the property that for every graph $G$ in it, it contains all induced subgraphs of $G$. Such classes are called hereditary. Many classes of theoretical and practical importance are hereditary, which includes, among others, planar, bipartite, split, threshold, perfect, interval, comparability, line graphs, forests, graphs of bounded vertex degree, etc. Many of those classes that are not hereditary have natural hereditary extensions: for instance, for the non-hereditary class of trees such an extension is the class of forests, and for the class of cubic graphs such an extension consists of all graphs of vertex degree at most three. Our interest in hereditary classes is based on the fact that these and only these classes admit a uniform description in terms of forbidden induced subgraphs. More formally, given a set of graphs $M$, let us denote by Free $(M)$ the class of graphs containing no induced subgraphs isomorphic to graphs in $M$. Then the following theorem holds.

Theorem 3.1 The class of graphs $X$ is hereditary if and only if $X=$ Free $(M)$ for a set $M$. Moreover, the minimal set $M$ with this property is unique.

Proof: Obviously, for any set $M$ the class $\operatorname{Free}(M)$ is hereditary. Conversely, let $X$ be a hereditary class, and $M$ the set of all minimal (with respect to the relation "to be an induced subgraph") graphs which are not in $X$. Clearly $X \subseteq$ Free $(M)$. On the other hand, every graph which is not in $X$ contains an induced subgraph from $M$. Therefore, $\operatorname{Free}(M) \subseteq X$. To prove the second part of the theorem, we will show that 
$M \subseteq N$ for any set $N$ such that $X=F r e e(N)$. To this end, let $G$ be a graph in $M$. By definition of $M, G$ does not belong to $X$, and hence, $G$ must contain an induced subgraph $H \in N$. By the same definition, every proper induced subgraph of $G$ belongs to $X$, from which we conclude that $G=H$, i.e. $G \in N$.

For many classes the induced subgraph characterization is known. For instance, according to the famous theorem of König [21], the class of bipartite graphs coincides with Free $\left(C_{3}, C_{5}, C_{7}, \ldots\right)$. Therefore, odd-chordality of bipartite graphs is 0 , while even-chordality is unbounded. For the larger class of comparability (or transitively orientable) graphs the induced subgraph characterization has been found by Gallai [11] (see also [10] and [26]). From this characterization it follows that odd-chordality of comparability graphs is at most 3 and even-chordality is unbounded. On the other hand, the same characterization shows that chordality of the complement of a comparability graph is at most 4 .

In general, the problem of finding induced subgraph characterization for a hereditary class might be very difficult, as the example of perfect graphs shows. However, for the purpose of our study, we do not need to know the complete list of minimal forbidden induced subgraphs. Indeed, with the above notation we can say that graphs in a class $X$ have chordality at most $k$ if $X \subseteq$ Free $\left(C_{k+1}, C_{k+2}, C_{k+3}, \ldots\right)$. Consider, for instance, the class of asteroidal triple-free (AT-free for short) graphs, which extends co-comparability graphs. In a graph, an asteroidal triple is a set of three pairwise non-adjacent vertices, any two of which are joined by a path avoiding the closed neigbhorhood of the third. Clearly any cycle with at least 6 vertices contains an asteroidal triple. Therefore, AT-free graphs constitute a subclass of Free $\left(C_{6}, C_{7}, C_{8}, \ldots\right)$, or equivalently, chordality of AT-free graphs is at most 5 , although the complete list of minimal forbidden graphs for this class is unknown (to our knowledge).

Below we propose a very simple sufficient condition for a class of graphs to have bounded chordality. The condition is based on the following helpful lemma.

Lemma 3.1 Free $\left(M_{1}\right) \subseteq$ Free $\left(M_{2}\right)$ if and only if every graph in $M_{2}$ contains a graph in $M_{1}$ as an induced subgraph.

Proof: Suppose first that a graph $H \in M_{2}$ does not contain induced subgraphs in the set $M_{1}$. Then $H \in \operatorname{Free}\left(M_{1}\right)-\operatorname{Free}\left(M_{2}\right)$, which proves necessity. Conversely, any graph $G \in \operatorname{Free}\left(M_{1}\right)-\operatorname{Free}\left(M_{2}\right)$ must contain an induced subgraph in $M_{2}$, and this graph cannot contain induced subgraphs belonging to $M_{1}$ (since otherwise $G \notin \operatorname{Free}\left(M_{1}\right)$ ). This proves sufficiency.

The following corollary is straightforward.

Corollary 3.1 Let $X=$ Free $(M)$ be a hereditary class of graphs. If $M$ contains a graph $G$ every connected component of which is a path, then chordality of graphs in $X$ is bounded. Specifically, if $k$ is the number of connected components of $G$ and $n_{j}$ is the number of vertices in the $j$-th component, then graphs in $X$ have chordality at most $\sum_{j=1}^{k} n_{j}+k-1$.

Now let us illustrate this simple statement with a number of examples.

1. (co- $\left.K_{n}\right)$-free graphs. The complement of a $K_{n}$ is the graph with $n$ isolated vertices. Therefore, by Corollary 3.1, chordality of (co- $\left.K_{n}\right)$-free graphs does not exceed $2 n-1$. Moreover, in the entire class of co- $K_{n}$-free graphs this bound is tight, since $C_{2 n-1}$ contains no complement of $K_{n}$ as an induced subgraph. However, for some specific subclasses of co- $K_{n}$-free graphs the bound can be further improved. Below we consider several such subclasses. 
1.1. Co-bipartite graphs. Co-bipartite graphs constitute a subclass of (co- $\left.K_{3}\right)$-free graphs and therefore, from the above general formula we conclude that their chordality cannot be more than 5. Furthermore, $C_{5}=\bar{C}_{5}$ is not a bipartite graph and hence chordality of co-bipartite graphs is at most 4 . This bound is tight, since $C_{4}$ is a co-bipartite graph. With further restriction to complements of $2 K_{2}$-free bipartite graphs (also known in the literature as difference graphs [19] or chain graphs [27]) we obtain a subclass of co-bipartite graphs of chordality at most 3 (the bound is tight).

1.2. Complements of graphs of vertex degree at most $d$. Clearly, a graph $G$ with maximum vertex degree at most $d$ is $K_{d+2}$-free. Therefore, the chordality of $\bar{G}$ is at most $2 d+3$. An improvement on this bound can be obtained by noticing that the complement of the graph $P_{d+1}+K_{1}$ (the disjoint union of $P_{d+1}$ and $K_{1}$ ) contains a vertex of degree $d+1$ and hence $P_{d+1}+K_{1}$ is forbidden in the class under consideration. Therefore, by Corollary 3.1, chordality of complements of graphs of vertex degree at most $d$ is bounded above by $d+3$. The bound is tight, since the complement of $C_{d+3}$ contains no vertices of degree greater than $d$.

1.3. Complements of graphs of degeneracy at most $k$. The degeneracy of a graph $G$ is the maximum value (over all induced subgraphs $H$ of $G$ ) of $\delta(H)$ where $\delta(H)$ is the minimum degree of $H$. Obviously, graphs of degeneracy at most $k$ are $K_{k+2}$-free. Let us show that their complements have chordality at most $k+3$. To this end, consider an induced cycle $C_{l}$ of length $l \geq k+4$. All vertices of $\bar{C}_{l}$ have degree $l-3 \geq k+1$. Therefore, cycles $C_{l}$ of length $l \geq k+4$ are forbidden for the class of complements of graphs of degeneracy at most $k$. The bound is tight, since an antihole on $k+3$ vertices is a regular graph with degree (and hence degeneracy) exactly $k$ and its complement is an induced $C_{k+3}$. Some examples of graphs of bounded degeneracy are those of bounded genus $g$, whose degeneracy and chordality are bounded below.

1.4. Complements of graphs of genus at most $g$. It is well-known that graphs of genus at most $g$ have at most $3 n-6+6 g$ edges. Using this, we claim that degeneracy of such graphs is at most $\sqrt{12 g}+3+\frac{9}{\sqrt{12 g}+3}$. To show this, consider a graph $G$ of genus $g$ and let $H=G[X]$ be any induced subgraph achieving the degeneracy $k$ of $G$. That is, $\delta(G[X])=k$.

Case 1: If $|X| \leq \sqrt{12 g}+3$, then $k \leq \sqrt{12 g}+3$.

Case 2: If $|X|>\sqrt{12 g}+3$, then since $H$ is also a genus- $g$ graph,

$$
k=\delta(H) \leq 6+\frac{12 g}{\sqrt{12 g}+3} \leq 6+\sqrt{12 g}-3+\frac{9}{\sqrt{12 g}+3}
$$

In any case, the degeneracy of $G$ is at most $\sqrt{12 g}+3+\frac{9}{\sqrt{12 g}+3}$.

Therefore, the chordality of $\bar{G}$ is at most $\sqrt{12 g}+6+\frac{9}{\sqrt{12 g}+3}$. For $g \geq 3$, the bound on chordality of complements of genus- $g$ graphs is tight up to an additive error of 3. To see this, consider an antihole $H$ on $m=k+3$ vertices where $k \geq 1$ is an integer. This is the same as a complete graph on $m$ vertices minus a hole on these $m$ vertices. It is well-known that a complete graph on $m$ vertices has genus exactly $(m-3)(m-4) / 12$. It follows that the genus $g$ of the antihole $H$ is at most

$$
\frac{(m-3)(m-4)}{12}=\frac{k^{2}-k}{12}
$$


Hence $\sqrt{12 g} \leq k-1+\varepsilon$ for some positive $\varepsilon<1$. Also, using $g \geq 3$,

$$
\sqrt{12 g}+6+\frac{9}{\sqrt{12 g}+3} \leq k+5+\varepsilon+\frac{9}{\sqrt{12 g}+3} \leq k+6+\varepsilon .
$$

This shows the tightness up to 3 . For $g=1,2$, the bound is tight up to an additive error of 4 .

1.5. Complements of graphs of bounded arboricity. The arboricity of a graph $G$ is the minimum number of edge-disjoint acyclic spanning subgraphs the union of which is $G$. According to Nash-Williams formula [23], the arboricity of $G$ coincides with $\max E(H) /(V(H)-1)$, where maximum is taken over all induced subgraphs $H$ of $G$. Therefore, graphs of bounded arboricity are $K_{n}$-free for some value of $n$, and thus complements of graphs of bounded arboricity have bounded chordality.

1.6. Complements of graphs in minor-closed classes. Graphs in minor-closed classes (i.e. those containing no graph in a certain family as a minor) have at most $c n$ edges [22], where $n$ is the number of vertices and $c$ is a constant associated with the class. Therefore, graphs in minor-closed classes have bounded arboricity and thus their complements are of bounded chordality. One of the most famous minorclosed classes is the class of planar graphs. Below we provide a tight bound for chordality of co-planar graphs.

1.7 Co-planar graphs. It is known that planar graphs have bounded degeneracy, genus, arboricity and they are $K_{5}$-free. Together with the above discussion this immediately leads to the conclusion that chordality of co-planar graphs is bounded. In order to derive a tight bound, let us first observe that co-planar graphs are $2 P_{3}$-free, since the complement of $2 P_{3}$ contains a $K_{3,3}$ as a subgraph. Therefore, by Corollary 3.1, chordality of co-planar graphs cannot be more than 7. To improve the bound, consider a cycle $C_{7}$ with vertices $a, b, c, d, e, f, g$ listed along the cycle. The complement of the cycle contains an edge subgraph $H$, which is homeomorphic to $K_{3,3}$ ( $H$ can be obtained by deleting the edges $a f, b d, e g, c e$ ). Hence chordality of co-planar graphs is at most 6 , and this bound is tight since the complement of an induced $C_{6}$ is planar.

2. Co-line graphs. The induced subgraph characterization of line graphs can be found, for instance, in [20]. One of the forbidden graphs for this class is the complement to $P_{2}+P_{3}$. Therefore, by Corollary 3.1 . chordality of co-line graphs is at most 6 . The bound is tight, since the complement of $C_{6}$ is a line graph (it does not contain forbidden graphs).

3. Co-chordal graphs are $2 K_{2}$-free and hence, by Corollary 3.1, their chordality is at most 5 . Moreover, since $C_{5}=\bar{C}_{5}$ is not a chordal graph, we conclude that chordality of co-chordal graphs is at most 4 . The bound is tight, since $2 K_{2}=\bar{C}_{4}$ is a chordal graph. Thus, we see that chordality is bounded both for chordal graphs and their complements, which is no wonder, since both classes are subclasses of weakly chordal graphs. By definition, a graph $G$ is weakly chordal if $G \in \mathrm{Free}\left(C_{5}, \bar{C}_{5}, C_{6}, \bar{C}_{6}, C_{7}, \bar{C}_{7}, \ldots\right)$. In addition to chordal graphs and their complements, the class of weakly chordal graphs contain many interesting subclasses, such as chordal bipartite [14], distance-hereditary [1], matroidal [24], tolerance graphs [18], etc. Therefore, all these graph classes and their complements have chordality at most 4.

\section{Conclusions}

In this paper we studied chordality of graphs in various classes. The main result is a proof of boundedness of even-chordality of co-circular-arc and co-circle graphs. There are many other important families of 
graphs for which the problem of determining chordality is open. In this section we discuss two of them. Both families are defined via an intersection model, both have numerous applications, and both generalize some known classes of graphs of low chordality, just as circular-arc and circle graphs. The first family is the class of circular permutation graphs [25]. Similarly to circle graphs, this is a generalization of permutation graphs. Chordality of permutation graphs, as well as their complements, is at most 4 , since this class is the intersection of comparability and co-comparability graphs. The other family was introduced in [17] under the name $k$-EPT graphs. This is a generalization of edge intersection graphs of paths in a tree (1-EPT graphs) [15] and vertex intersection graphs of paths in a tree (VPT graphs) [16]. Every VPT graph is chordal, since chordal graphs are exactly the vertex intersection graphs of subtrees of a tree [13]. Therefore, chordality is bounded both for VPT graphs and their complements. The class of 1-EPT graphs is an extension of VPT graphs. Chordality of 1-EPT graphs is unbounded, while co-chordality (i.e. chordality of their complements) is at most 6 [15]. $k$-EPT graphs constitute a further generalization of both classes, and therefore, provide a new direction for future research.

\section{References}

[1] H.-J. Bandelt and H.M. Mulder, Distance-hereditary graphs, J. Combinatorial Theory B 41 (1986) 182-208.

[2] H. L. Bodlaender, A tourist guide through treewidth, Acta Cybernetica, 11 (1993), pp. 1-21.

[3] H. L. Bodlaender And D. M. Thilikos, Treewidth for graphs with small chordality, Discrete Applied Mathematics, 79 (1997), pp. 45-61.

[4] V. ChepoI and F. DRAGAN, A note on distance approximating trees in graphs, European J. Combinatorics, 21 (2000) 761-766.

[5] L. S. ChandRAN AND L. S. RAm, On the number of min-cuts in a graph, in Proceedings of the 8th International computing and combinatorics conference, LNCS 2387, 2002, pp. 220-230.

[6] L. S. ChANDRAN AND C. R. SubRAmANiAn, A spectral lower bound for the treewidth of a graph and its consequences, Information Processing Letters, 87 (2003), pp. 195-200.

[7] M. Chudnovsky, N. Robertson, P. Seymour and R. Thomas, The Strong Perfect Graph Theorem, manuscript, June 2003.

[8] K. DEYMER, A new apper bound for the length of snakes, Combinatorica 5 (1985) 109-120.

[9] F.F. DRAGAN, Estimating all pairs shortest paths in restricted graph families: a unified approach, Journal of Algorithms, accepted.

[10] P. DucheT, Classical perfect graphs: an introduction with emphasis on triangulated and interval graphs, Annals of Discrete Mathematics, 21 (1984) 67-96.

[11] T. Gallai, Transitiv orientierbare Graphen, Acta Math. Acad. Sci. Hung. 18 (1967) 25-66.

[12] F. GAVRIL, Algorithms for maximum weight induced paths, Information processing Letters, 81 (2002) 203-208. 
[13] F. GAVRIL, The intersection graphs of subtrees in trees are exactly the chordal graphs, J. Combinatorial Theory B, 16 (1974) 47-56.

[14] M.C. GolumbiC and C.F. Goss, Perfect elimination and chordal bipartite graphs, J. Graph Theory, 2 (1978) 155-163.

[15] M.C. GolumbiC and R.E. JAmison, The edge intersection graphs of paths in a tree, J. Combinatorial Theory B, 38 (1985) 8-22.

[16] M.C. GolumbiC and R.E. JAmison, Edge and vertex intersection of paths in a tree, Discrete Mathematics, 55 (1985) 151-159.

[17] M.C. Golumbic, M. LiPShTEYn and M. STERn, The recognition of k-EPT graphs, Congressus Numerantium, 171 (2004) 129-139.

[18] M.C. Golumbic and A.N. Trenk, Tolerance graphs Cambridge Studies in Advanced Mathematics, 89. Cambridge University Press, Cambridge, 2004. xii+265 pp.

[19] P.L. Hammer, U.N. Peled, and X. Sun, Difference graphs, Discrete Applied Mathematics, 28 (1990) 35-44.

[20] F. HARRARY, Graph Theory, Addison-Wesley, Reading, MA, 1969.

[21] D. KöNIG, Über Graphen und ihre Anwendungen auf Determinantentheorie und Mengenlehre, Math. Annal. 77 (1916) 435-465.

[22] A.V. Kostochка, Lower bound of the Hadwiger number of graphs by their average degree, Combinatorica, 4 (1984) 307-316.

[23] C. NASH-Williams, Edge-disjoint spanning trees of finite graphs, J. London Math. Soc. 36 (1961) 445-450.

[24] U.N. Peled, Matroidal graphs Discrete Math. 20 (1977/78) 263-286.

[25] D. Rotem and J. Urrutia, Circular permutation graphs, Networks, 12 (1982) 429-437.

[26] W.T. Trotter, Combinatorics and partially ordered sets. Dimension theory. Johns Hopkins Series in the Mathematical Sciences. Johns Hopkins University Press, Baltimore, MD, 1992. xvi+307 pp.

[27] M .YAnNAKAKIS, Node-deletion problems on bipartite graphs, SIAM J. Computing 10 (1981) 310-327. 
